\title{
La Asociación Alonso Quijano de Madrid: participación social en la salud mental
}

\author{
Juan Carlos Casal Álvarez \\ Asociación Alonso Quijano, Madrid
}

\begin{abstract}
Resumen
Este artículo recoge las experiencias personales y asociativas con la Asociación Alonso Quijano de Madrid que he vivido desde el año 2002. La Asociación Alonso Quijano está inscrita en el registro de Asociaciones de la Comunidad de Madrid, se fundó en el año 1999. La Asociación Alonso Quijano es una Asociación para acompañar, apoyar y reflexionar en la experiencia de la locura. Desde su fundación el rol que asumen los participantes es el de ciudadanos con los mismos derechos y obligaciones y sin poner etiquetas. Es una asociación donde la participación de las personas con problemas de salud mental es muy relevante.
\end{abstract}

Palabras claves: salud mental; diversidad funcional; participación social; ayuda mutua; ocio organizado.

\begin{abstract}
The Alonso Quijano of Madrid Association: social participation in mental health. This article, brings together, the individual and collective experiences that I had the opportunity to experience with the Alonso Quijano of Madrid Association since 2002. The Alonso Quijano Association is registered in the Official Association Register of the Madrid Local Government, and was established in 1999. Alonso Quijano Association is an association to monitor, support and reflect on the experience of madness. Since its origins, the members asume the rol of citizens with the same rights and obligations without labelling anybody. It is an association where the involvement of people with mental health problems is very important.
\end{abstract}

Keywords: mental health; functional diversity; social participation; mutual help; organized leisure time.

$\mathrm{L}$ a Asociación Alonso Quijano está inscrita en el registro de Asociaciones de la Comunidad de Madrid y esta declarada de utilidad pública municipal por el Ayuntamiento de Madrid. El número de socios es de setenta personas. Se fundo en el año 1999 por un grupo de antiguos pacientes, familiares y profesionales del Hospital de Día Madrid ${ }^{1}$. Desde su fundación el rol que asumen los participantes es el de ciudadanos con los mismos derechos y obligaciones y sin poner etiquetas. Es una asociación donde la participación de las personas con problemas de salud mental es muy relevante.

\section{Los participantes}

Todas las personas que participan con la asociación Alonso Quijano han tenido experiencias con la locura y la mayoría las han vivido en primera persona. Los problemas de salud mental a veces nos impiden ser felices, trabajar, estudiar e incluso hacer las actividades básicas de la vida diaria. Nos cuesta estar motivados, tener iniciativa y mantener las actividades. Nos lleva a perder relaciones sociales, nos lleva al aislamiento no deseado. Tenemos síntomas de angustia, alucinaciones, delirios, pensamientos obsesivos y conductas impulsivas. Padecemos de obesidad, diabetes y de hipertensión. Tenemos abundantes conflictos familiares. Los problemas de salud mental hacen que llevemos complejos y largos tratamientos psicoterapéuticos y farmacológicos. Conocemos los efectos secundarios de los fármacos y la historia atroz de exclusión, reclusión y exterminio que han sufrido y que aún sufren los enfermos mentales privados de libertad y/o del derecho a vivir en comunidad. Somos usuarios de los centros de salud mental y de los servicios sociales especializados para la rehabilitación psicosocial de las personas con enfermedad mental grave y crónica.

La mayoría tenemos un grado de discapacidad superior al $33 \%$. y muchos con más de un $65 \%$ de grado de discapacidad (Boletín Oficial del Estado, 2003) lo que nos da derecho a reclamar del estado una pensión no contributiva de escasa cuantía o nuestros familiares cobran la pensión por hijo a cargo. Nuestra esperanza de vida es diez años más corta que la de las otras personas sin problemas de salud mental, algunas personas son muy dependientes de sus familiares pero no entran en los baremos de la ley de dependencia (Boletín Oficial del Estado, 2006) para tener ayudas públicas. Muchos viven con sus padres mayores que necesitan cuidados pero siguen siendo cuidadores (Muñoz, Pérez-Santos, Crespo, \& Guillen, 2005). Se nos llama de muchas maneras: personas con enfermedad mental, usuarios, personas con enfermedad mental grave y crónica, supervivientes de la psiquiatría, discapacitados psicosociales, personas con 
diversidad funcional mental ${ }^{2}$, personas con trastorno mental severo. No tenemos espacios de encuentro, de reunión ni físicos ni virtuales. Las asociaciones de nuestro colectivo están demasiado dispersas y centradas en la petición de la subvención para ofrecer servicios para unos pocos socios, basados en el esfuerzo de voluntarios y profesionales mal pagados y que deberían ser asumidos por los sistemas de sanidad y servicios sociales de las administraciones.

\section{El estigma y el aislamiento son barreras que hay que eliminar con acción social}

El estudio de la salud y sus determinantes se facilita mediante representaciones formales llamadas modelos. E1 modelo de la salud denominado de los determinantes de la salud, conceptúa la salud como algo más que la simple ausencia de enfermedad y como resultado de la integración de un conjunto de factores que pueden agruparse en cuatro grandes categorías: los estilos de vida, el sistema de cuidados de salud, la propia biología humana y el medio ambiente. Entre los determinantes de la salud se incluyen factores como el estrés, la hostilidad, la clase social o el apoyo de las personas que nos rodean. En los problemas de salud mental son un problema añadido muy importantes los prejuicios hacia la locura y hacia el colectivo de personas con problema de salud mental, el estigma junto con la restricción en la participación social y la red social de apoyo. Formulando estrategias y métodos de intervención sobre estos determinantes podremos promocionar la salud y provocar un cambio social (Álvarez-Dardet \& Colomer, 2006).

La discriminación es un hecho evidente, como resalta el informe sobre la panorámica de la discapacidad en España. De la Encuesta de Discapacidad, Autonomía personal y situaciones de Dependencia (Instituto Nacional de Estadística, 2008):

La deficiencia que causa mayor número de discapacidades por persona es la mental... El menor porcentaje de población ocupada está entre los colectivos que tenían limitaciones de aprendizaje, aplicación de conocimientos, desarrollo de tareas y de interacciones y relaciones personales. El perfil de la persona que se siente discriminada es un varón, menor de 65 años, desempleado, con deficiencias mentales o del sistema nervioso y con dificultad en sus relaciones personales (p. 8).

Aunque todos tenemos los mismos derechos, aún hay un notable déficit de participación social y de acceso a los apoyos necesarios para que la población afectada en general pueda disfrutar también de una vida autónoma e independiente.

\section{Nuestro convencimiento}

Todos los ciudadanos y ciudadanas diagnosticados o no y de cualquier condición y profesión tenemos que luchar activamente por la defensa de los derechos humanos y contra los prejuicios del estigma de la enfermedad mental. Las personas con problema de salud mental tenemos una responsabilidad añadida con nuestro propio proceso de cambio y de crecimiento personal. Retomar el propio curso vital y recuperar al máximo las propias capacidades como individuo y como ciudadano. Responsabilidad y participación como expertos en nuestro problema de salud mental. Participación y responsabilidad en la promoción y producción de salud. Participación y responsabilidad para estar representados a todos los efectos en cualquier foro, o agencia donde se hable de nosotras y nosotros (Lobato \& Romañach, 2005). Estamos convencidos de que las personas con problemas de salud mental tienen que tener lugares de reunión para el debate e intercambio de ideas y donde el estigma de la enfermedad mental no esté presente (Palacios \& Bariffi, 2007). Hemos creado una red informal de ciudadanos interesados por los problemas de la salud mental. Mantenemos dos grupos que funcionan como grupos de ayuda mutua, uno orientado para los familiares más allegados y otro grupo para las propias personas con problemas de salud mental. A este grupo lo denominamos club social. También organizamos certámenes de poesía y encuentros musicales con el tema central de la locura.

\section{La participación en grupos de ayuda mutua ${ }^{3}$ facilita la recuperación y la integración social.}

La participación de las personas con enfermedad mental en la promoción y producción de salud es clave. No puede ser sustituida ni por la familia, ni por los profesionales de la salud mental, ni por los servicios de salud. A todos nos corresponde comportarnos fraternalmente y prestarnos apoyo mutuo. Ser personas activas en la verdadera integración y no exclusión del colectivo y de cada persona, evitar el aislamiento de nuestros compañeros y del colectivo. Mostrar una imagen diferente de las personas con problemas de salud mental y combatir con todos los medios a nuestro alcance la falta de respeto a cualquier ciudadano por motivos de salud mental. Todos los ciudadanos y ciudadanas diagnosticados o no y de cualquier condición y profesión tenemos que luchar activamente por la defensa de los derechos humanos y contra los prejuicios del estigma de la enfermedad mental (Federación Española de Asociaciones de Rehabilitación Psicosocial [FEARP], 2006) ${ }^{4}$.

Una forma eficaz de mejora de la calidad de vida y de ejercicio real de los derechos de ciudadanía es la práctica de la ayuda mutua. La participación activa en las asociaciones y en particular la práctica de ayuda mutua favorece la adherencia a los tratamientos, mejora la calidad de vida de las personas con enfermedad mental. Aumenta la red social de la persona afectada y de sus familias. Hacen a la persona más autónoma, responsable y solidaria. Aumentan las oportunidades de participación social, integración laboral, cultural y de ocio organizado. Se pueden buscar nuevas formas de convivencia (Palacios \& Romañach, 2006). En nuestra asociación mantenemos desde hace más de diez años dos grupos de ayuda mutua el Grupo de familias y el Club Social y disponemos de una página Web:

www.a-alonsoquijano.org y de un blog http://aalonsoquijano.blogspot.com/ para dar a conocer nuestra actividades.

\section{Escuela de Familias, grupo de Padres}

El Grupo de Familias AAQ se formó en 2001, desde entonces han participado en él unas 50 familias. El grupo de familias esta dirigido a miembros del grupo de convivencia (familiares, padres, madres, tutores, acogedores) de personas que han sufrido crisis psiquiátricas que han interrumpido o alterado el curso de sus vidas y modificado las rutinas de la convivencia. Es 
un grupo de apoyo mutuo, dedicado a cuidar de los cuidadores, sabiendo que ello va a redundar en la mejoría de la situación y el bienestar de todos (usuarios y miembros del entorno).

La aparición en cualquiera de los miembros de una familia de una crisis o enfermedad psiquiátrica, interrumpe la continuidad de la vida familiar y supone para todos los que conviven un verdadero cataclismo emocional. Muy habitualmente surgen prejuicios, inquietudes, miedos, ansiedades, preguntas por la causa y dudas sobre como afrontar la situación, temores de todo tipo, irracionales sentimientos de culpa, dudas por el futuro y toda clase de incertidumbres, que son muy difíciles de afrontar en solitario.

Las familias corren el riesgo de sentirse solas, aisladas e incomprendidas, afrontando solas los prejuicios y el estigma social que compaña a la enfermedad y de des-estructurarse como consecuencia del problema. El objetivo de este grupo es poder hablar, compartir ideas e impresiones, para vencerlos, acompañarse en el recorrido, descubrir que no tiene sentido buscar culpables, apoyar y experimentar las oportunidades que ofrece el apoyo mutuo en este difícil viaje en el que todos tienen cosas que aportar. Existe evidencia médica que sugiere que la participación en grupos de familia de apoyo mutuo mejora el pronóstico del curso del enfermo, y el bienestar y ajuste de la familia a la nueva situación.

\section{Club Social}

El Club Social es un grupo abierto que se reúne desde el año 1997. Un grupo de jóvenes que habían sido dados de alta del Hospital de Día Madrid, expresan su voluntad de seguir quedando. Con el apoyo de profesionales que contribuyen a facilitar un espacio de encuentro, estabilidad y permanencia de las reuniones semanales.

En el Club Social trabajamos la capacidad y la autonomía de la persona para llevar adelante el proyecto vital personal. Son relaciones reales de interacción e interdependencia en un grupo humano basado en el respeto, la tolerancia, la solidaridad, el apoyo entre iguales. Hacemos que mejoré la calidad de vida de los compañeros de asociación y de otras personas interesadas dedicando de forma altruista parte de nuestro tiempo de ocio. El club social es una herramienta que usa la interacción comunitaria y potenciación de habilidades sociales para promover bienestar social y ganancia de salud. Es un grupo abierto de ciudadanos con muchas ganas de vivir y mejorar nuestras vidas junto a otros que conocemos y que queremos conocer. Con mucha interacción, relaciones entre nosotros y con otras personas interesadas, usuarios, familiares y profesionales de la salud mental.

Durante el año son convocadas a las actividades una media de 30 personas. Los hombres y mujeres de edades entre los $18 \mathrm{y}$ los 60 años que formamos parte de ese grupo tenemos diferentes profesiones, diversos diagnósticos, discapacidades, algunos con patología dual, algunas personas son personas tuteladas, somos personas afectadas directamente por los problemas que conllevan los procesos de salud mental y allegados (familiares, amigos, profesionales de la salud.). Con trabajo, en el paro, jubilados, pensionistas con diferente afectación y fase de recuperación o persistencia de los síntoma de enfermedad. Somos ciudadanos con residencia en la Comunidad de Madrid.
Es un grupo en el que las personas tienen "tantas etiquetas" que nos definimos como ciudadanos y ciudadanas. Se fomenta el respeto y la tolerancia hacia los demás, se tiene en cuenta a los participantes como personas con nombre y apellidos y no como personas etiquetadas. Ninguno de los miembros desempeña un papel más importante que otro. No todas las personas acuden a todas las reuniones, algunos días somos cuatro y otros más de veinte. El compromiso con las actividades del grupo es personal y variable. Hay participantes que llevan asistiendo a las reuniones desde su fundación. Algunos de nosotros actuamos como moderadores y facilitadores del encuentro, este es un compromiso que se asume por periodos anuales. Estas personas ejercen un papel de liderazgo en el grupo.

Destacando que en la actualidad hay un médico-terapeuta, dos psicólogos, un trabajador social que están muy familiarizados por diferentes motivos y no solo por su profesión por los problemas que afectan a los integrantes del grupo y al grupo. Los profesionales de la salud que participan no tienen una función directiva, si ejercen un papel técnico en los momentos que hay que resolver algún conflicto puntual.

La reunión formal dura unas dos horas y media a la semana, aunque nuestra relación no se ciñe a este horario. La primera media hora la dedicamos a saludarnos, contar como ha ido la semana, acoger a alguna persona nueva o que hace tiempo que no vemos. Después y durante una hora realizamos una actividad (esta actividad salvo excepciones no esta programada, ni planificada) y puede ser, una charla distendida sobre temas de actualidad, la marcha del grupo, temas asociativos. Derechos y recursos sociales en nuestro entorno. Temas personales. Juegos de mesa, video forum. Organizamos actividades de ocio que se pueden realizar en fin de semana o durante la reunión semanal. Efectuamos evaluaciones periódicas de lo que vamos realizando y de las nuevas propuestas. De lo que menos hablamos es de enfermedad mental. Después nos vamos a un bar cercano para seguir charlando y tomar algo. Nos vamos despidiendo según el tiempo disponible que cada uno tenga.

Más allá de la reunión semanal, el apoyo entre nosotros se extiende a otros espacios y a otros días de la semana, nos reunimos solo con unas normas básicas que están incluidas en el reglamento de la Asociación: Todos los participantes tienen la obligación de respetar las convicciones políticas, morales religiosas de las personas integrantes del grupo. $\mathrm{Y}$ todos los participantes se obligan a observar una conducta basada en el mutuo respeto, tolerancia y colaboración encaminada a facilitar una mejor convivencia.

\section{El certamen de poesía}

Una forma de combatir la estigmatización social que previenen a la sociedad de la relación con las personas enfermas debido a prejuicios falsos e injustos es la convocatoria periódica de certámenes de poesía y actos musicales. Apoyando la dignidad de las personas, el tratamiento normalizado de la enfermedad y la correcta información a la sociedad sobre las condiciones de la vida de las personas, especialmente si pueden difundir informaciones positivas que alienten la normalización y la esperanza en una vida digna y participativa.

La convocatoria desde la asociación del certamen de 
poesía permite la participación de personas con problemas de salud mental. Las personas se sentirán apoyadas para expresarse y desafiar los estereotipos sociales discriminatorios y estigmatizantes. Enviamos un mensaje positivo a la sociedad, para que contemple la enfermedad desde el punto de vista de las producciones literarias de los afectados y de otras personas sensibilizadas. Y además produce material positivo para los medios de comunicación social (prensa y radio), que ayudaran a mejorar la imagen social de las personas afectadas, reforzando la confianza en los medios asociativos para futuro proyectos. Hemos convocado tres certámenes poéticos internacionales Asociación Alonso Quijano de Madrid y editado dos libros de poemas, "Vesanias" y "Locos por la Vida".

\section{En proceso de cambio}

Desde hace cuatro años somos más participativos y tenemos muchas actividades de ocio organizado. Hacemos viajes aprovechando los programas del IMSERSO, hemos realizado cuatro, estuvimos en Praga, Lisboa y dos viajes a las islas canarias. Es una forma de tener vacaciones, muchos de nosotros no tenemos oportunidad de realizar viajes con nuestra familia u otros amigos. Organizamos un grupo de unas diez a doce personas y nombramos entre nosotros dos monitores. Es una experiencia muy gratificante y nuestros familiares cuidadores aprovechan también para tener sus vacaciones. Hace dos años que quedamos los fines de semana para ver exposiciones, ir al cine escuchar música, salir a cenar y tomar un café, preparamos excursiones por la montaña. Para nosotros es muy importante tener actividades de tiempo libre, muchos de nosotros no trabajamos, ni estudiamos pero si podemos disfrutar de la cultura y de la naturaleza.

Recientemente hemos trasladado la sede del club social al barrio de Lavapiés. La sede legal de Asociación esta en un distrito periférico de la ciudad de Madrid (Ciudad Lineal) y los grupos se reúnen en locales cedidos. El grupo de familias en un local cedido por una entidad privada y el Club Social en el Centro Comunitario del Casino de la Reina, un equipamiento de servicios sociales del Ayuntamiento de Madrid en el distrito Centro de Madrid. Queremos estar presentes en un barrio donde la diversidad cultural y la acción ciudadana son palpables. Hace tiempo que entramos en contacto con el Centro de Día y el Equipo de Apoyo Comunitario de este lugar castizo. Cada día acuden a conocernos personas nuevas del barrio y estamos mas cercas de la oferta cultural y de ocio de la ciudad de Madrid.
Tenemos mas oportunidades de ocio en la ciudad y de entrar en contacto con otros grupos que también realizan actividades en el barrio.

También queremos unirnos a otros grupos para luchar a favor de la salud mental de Madrid como la Federación Madrileña de Asociaciones de Salud Mental (FEMASAM) ${ }^{5}$, para tener una mayor información y nuevas oportunidades. Este año 2011 vamos a convocar el IV Certamen Poético Internacional de la Asociación Alonso Quijano de Madrid lo que nos ayudara a darnos a conocer en el distrito centro y presentarnos a otros grupos. La Asociación Alonso Quijano es una Asociación para acompañar, apoyar y reflexionar en la experiencia de la locura.

\section{Referencias}

Federación Española de Asociaciones de Rehabilitación Psicosocial (2006). Modelo de Atención a Personas con Enfermedad Mental Grave (Documento de Consenso). Madrid: Ministerio de Trabajo y Asuntos Sociales.

Instituto Nacional de Estadística (2008). Panorámica de la discapacidad en España. Informe Encuesta sobre Discapacidades, Autonomía personal y situaciones de Dependencia. Madrid: Instituto Nacional de Estadística.

Ley n. 51, de 2 di diciembre de 2003 (2003, 2 di diciembre). De igualdad de oportunidades, no discriminación y accesibilidad universal de las personas con discapacidad. Espanã, Jefatura del Estado, Boletín Oficial del Estado, 289, de 3 di diciembre de 2003.

Ley n. 39, de 14 di diciembre de 2006 (2006, 14 di diciembre). de Promoción de la Autonomía Personal y Atención a las personas en situación de dependencia. Espanã, Jefatura del Estado, Boletín Oficial del Estado, 299, de 15 di diciembre de 2006.

Muñoz, M., Pérez-Santos, E., Crespo, M., \& Guillen, A. I. (2005). Resumen de Resultados de la investigación: el estigma de la enfermedad mental. Madrid: Universidad Complutense de Madrid.

Palacios, A., \& Bariffi, F. (2007). La discapacidad como una cuestión de Derechos Humanos. Una aproximación a la Convención Internacional sobre los derechos humanos de las personas con Discapacidad. Colección telefónica accesible. CERMI. Madrid: Editorial Cinca.

Palacios, A., \& Romañach, J. (2006). El modelo de la diversidad. La Bioética y los Derechos Humanos como herramientas para alcanzar la plena dignidad en la diversidad funcional. Madrid: Diversitas Ediciones.

Revuelta, C. C., \& Díaz, C. Á.-D. (2006). Promoción de la saludy cambio social. Barcelona: Editorial Elsevier-Masson.

Romañach, J., \& Lobato, M. (2005). Diversidad Funcional nuevo término para la lucha por la dignidad en la diversidad Humana. Foro de vida Independiente, 1-8. Recuperado de http://www.minusval2000.com/ relaciones/vidaIndependiente/diversidad_funcional.html. 
1. Se trata de un dispositivo sanitario específico para usuarios graves que ofrece, en régimen de hospitalización parcial, tratamientos intensivos, durante el tiempo preciso en cada caso, destinados a promover un cambio en el funcionamiento psicológico del usuario que permita la continuidad de seguimiento y tratamiento ambulatorio en su Servicio de Salud Mental: http://www.hdmadrid.org

2. http://www.forovidaindependiente.org/ Término acuñado por el Foro de Vida Independiente.

3. La ayuda mutua es una práctica de intercambio que se establece entre dos o más personas y que repercute positivamente sobre los que la practican.

4. El Instituto de Mayores y Servicios Sociales- El Imserso - es la Entidad Gestora de la Seguridad Social para la gestión de los Servicios Sociales complementarios de las prestaciones del Sistema de Seguridad Social, y en materia de personas mayores y personas en situación de dependencia. http://www.imserso.es.

5. La Federación Madrileña de Asociaciones Pro Salud Mental (FEMASAM) integra el movimiento asociativo que representa a familiares, amigos y a todas aquellas personas interesadas en defender los intereses de los ciudadanos que, fundamentalmente en la Comunidad de Madrid, padecen trastornos mentales severos y persistentes. http://www. femasam.org.

Juan Carlos Casal Álvarez é estudante de Trabajo Social, presidente de la Asociación Alonso Quijano de Madrid e Coordenador do Club Social. Endereço para correspondencia: C/ la Bañeza nº 10, 8º. 28029, Madrid.E-mail: jccasal@gmail.com 